\title{
Performance analysis of realistic optical time division multiplexed wavelength-routed networks
}

\author{
C. Y. $\mathrm{Li}^{\dagger}{ }^{\dagger}$ G. M. Li ${ }^{\ddagger}$ P. K. A. Wai, ${ }^{\dagger}$ and V. O. K. $\mathrm{Li}^{\ddagger}$ \\ ${ }^{\dagger}$ Photonics Research Center and Department of Electronic and Information Engineering, \\ The Hong Kong Polytechnic University, Hong Kong \\ Tel: +852 2766-4094, Fax: +852 2362-8439, E-mails: \{enli, enwai\}@ polyu.edu.hk \\ $\ddagger$ Department of Electrical and Electronic Engineering, The University of Hong Kong, Hong Kong \\ Tel: +852 2857-8425, Fax: +852 2559-8738, E-mails: $\{$ gmli, vli $\} @ e e . h k u . h k$
}

\begin{abstract}
Application of optical time division multiplexing (OTDM) in wavelength routed optical networks will greatly enhance the flexibility of bandwidth assignment because OTDM provides time division sub-channels in a wavelength to match the processing speed of electronic devices. Different types of such OTDM wavelength-routed (OTDM-WR) networks assuming different level of sophistication of the OTDM technology have been proposed. The performance of these OTDM-WR networks improves with the time-slot routing capability of the intermediate nodes of the network. However, as the transmission rate increases up to hundreds of gigabits per wavelength channel, electronic processing of the time slots limit the achievable performance of the OTDM-WR networks. All-optical signal processing can overcome the electronics bottleneck but available all-optical signal processing capability is rather limited and cannot utilize the full potential of time-slot routing; for example, all-optical time slot interchange is not practical yet. Even with such limitations, current technologies such as fast wavelength converters and micro-electromechanical system (MEMS) optical switches can significantly enhance the performance of existing wavelength-routed networks by adding the OTDM capability, albeit limited. In this paper, we develop time-slot routing schemes that requires fast wavelength converters only and study the performance of these schemes by simulations on Manhattan street networks and a network of topology of AT\&T North America OC-48 fiber network.
\end{abstract}

\section{INTRODUCTION}

Wavelength-routed (WR) optical networks are one of the important networking platforms for providing broadband services [1]. In WR networks, wavelength channels are used for the communication between the source and the destination nodes. After setting up wavelength channels, data streams remain in optical format until they arrive at the destination nodes. Intermediate nodes route the data according to the incoming ports and the associated wavelengths of the data streams. The complexity of routing is reduced to minimum and no electronic processing is required at intermediate nodes. Transmission rate up to tens of gigabits per second on a wavelength channel is commonly available in WR networks. The maximum transmission

This research is supported in part by the Areas of Excellence Scheme established under the University Grants Committee of the Hong Kong Special Administrative Region, China (Project No. AoE/E-01/99). Additional support is provided by a grant from The Hong Kong Polytechnic University (Project Number A-PD91). rate is mainly determined by the data processing capability of the source and destination nodes. Since users are guaranteed their transmission bandwidth once they get connected, blocking is the main performance indicator in WR networks [1].

The probability of successful wavelength channel provision depends on factors such as the wavelength channel assignment mechanisms, the routing algorithms, the availability of wavelength converters, and the allowed number of wavelength channels per link. In general, WR networks using wavelength channel assignments that use network status information can serve more users than fixed assignments. Adaptive routing algorithms utilize wavelength channels more efficiently than fixed routing [2]. Wavelength converters improve the availability of wavelength channels by allowing the channels to be made up of segments with different wavelengths [1]. All these methods optimize the utilization of the limited number wavelength channels in WR networks.

Optical time division multiplexing (OTDM) can enhance the flexibility of bandwidth assignment in WR networks [3]. OTDM provides time division sub-channels in a wavelength channel to match the processing speed of electronic devices, and allows users to access the sub-channels individually. Users can have better chance of being served in OTDM-WR networks because multiple users can share the same wavelength channel. Different types OTDM-WR networks based on time-shared space switching technology have been proposed [4]-[7]. In time-shared space switching a data slot at an input port of a node can be switched to any output port but the wavelength and time division sub-channel of the data remain unchanged. As the transmission rate increases up to hundreds of gigabits per wavelength channel, the routing at immediate nodes will have to be carried out all-optically. Currently available optical signal processing power however is rather limited, for example, neither all-optical time slot interchangers nor large time-shared space switches are available yet. Thus the full potential of time division switching cannot be utilized. However, we observe that current technologies such as wavelength converters [8] and micro-electro-mechanical system (MEMS) optical switches [9] can already significantly improve the performance of existing wavelength-routed networks. 
In this paper we investigate the performance of OTDM-WR networks constructed with fast wavelength converters only. No time-shared space switching is assumed. We show that the performance of the proposed networks is comparable to those with time-shared space switching only and much better than wavelength routed networks. Thus one can add OTDM to WR networks using present day technology. The paper is organized as follows. In Section II we review the time division switching in OTDM-WR networks, and discuss the difficulties of all-optical implementation. The proposed wavelength-switched time-slot routing scheme is then described in Section III. We compare the performance of different kinds of time-slot routing schemes by simulations in Section IV. We demonstrate that the proposed time-slot routing scheme can provide significant performance improvement on current WR networks. Finally, we give the conclusion in Section V.

\section{OPTICAL TIME DIVISION MULTIPLEXED WAVELENGTH-ROUTED NETWORKS}

OTDM-WR networks are connection-oriented networks. A user requests for a connection by sending the destination address and the required quality of service such as transmission bandwidth to the network. The network then searches for a suitable path to fulfill the request. If a path is found, one or more time division wavelength sub-channels at each link on the path are reserved for the user. The user is granted the right to send out his data to the assigned wavelength channels within the particular time slots of the periodic time frames. The path searching algorithm may determine the path by looking up precomputed records [4]-[5], or computing the path using current information of the network status [7]. The permitted time for path searching and time division sub-channel reservation is in general sufficiently large such that electronic processing can be used. Once the sub-channel reservation process is completed, intermediate nodes lookup the routes according to the input ports, the wavelength channels, and the time division subchannels associated to the user data. Because of the simplicity, the route lookup at intermediate nodes can be processed very fast even if optical processing is not used. However, the switching of data from the input ports to the output ports has to be done all-optically to avoid conversion of the data between the optical domain and the electrical domain which will significantly slow down the switching process.

The requirement for all-optical switching of data and the limited optical signal processing power renders some traditional time division switching approaches unfeasible in OTDM-WR networks. For example, optical time slot interchange is not possible yet. However, features unique to optical networks such as wavelength conversion/switching allow new functions of time division switching to be developed in OTDM-WR networks. We may manipulate data with time division switching in the wavelength domain in addition to the time domain and space domain. Time-slot routing schemes in OTDM-WR networks

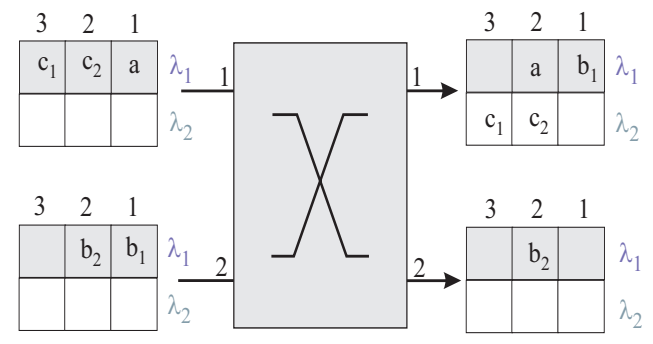

Fig. 1. An optical time division multiplexed wavelength-routed (OTDM-WR) node with routing processing in space, time, and wavelength domains. The node has two input ports and two output ports. Two wavelength channels $\left(\lambda_{1}\right.$ and $\lambda_{2}$ ) are available for each input/output port. Each wavelength channel is further divided into three time division sub-channels.

can be very different if different considerations and assumptions on the optical processing power in the following three switching categories are assumed.

\section{A. Time switching}

In traditional time division switching networks, one is able to arbitrarily modify the time division sub-channel allocation of a data with time slot interchangers (TSI) [10]. As shown in Fig. 1, data $a$ is switched from slot 1 of input port 1 to slot 2 of output port 1 with TSIs being installed on both input ports. TSIs are one of the key devices in traditional time division switching networks, but TSIs for optical networks are not available yet because of the lack of practical optical buffer and sophisticated optical processing devices. Most proposed time-slot routing schemes do not assume TSIs in OTDM-WR networks.

\section{B. Space switching}

Time-shared space switching is another powerful switching mechanism in traditional time division switching networks [10]. Data in adjacent time slots in a data stream at the input port of a node can be switched to different output ports. For example, data $b_{1}$ and $b_{2}$ from input port 2 in Fig. 1 are switched to different output ports but their associated wavelengths and time division sub-channels must remain unchanged as $\left(\lambda_{1}, 1\right)$ and $\left(\lambda_{1}, 2\right)$, respectively. Time-shared space switching is traditionally used with TSIs to build large switches in time division switching networks. It is not recommended to build switches with only time-shared space switching because the blocking probability will be large [10], e.g., data $b_{1}$ and $b_{2}$ in Fig. 1 cannot be switched correctly if data $a$ has not been moved to time slot 2 at output port 1 . In addition, fast optical switches are available in small size only, e.g., $2 \times 2$. In spite of this, timeshared space switching is commonly assumed to be available in OTDM-WR networks in the derivation of time-slot routing schemes [4]-[7].

\section{Wavelength switching}

Although wavelength division multiplexing (WDM) greatly simplifies bandwidth utilization and data routing in optical networks, WDM occasionally under-utilizes the system because 
fiber bandwidth is divided into non-overlap channels. Data streams in different wavelength channels do not interfere with each other but sharing of bandwidth is also prohibited. Wavelength conversion overcomes this by allowing data streams to change their wavelength channels at intermediate nodes to improve the bandwidth utilization [8]. Similarly, wavelength conversion reduces the blocking in OTDM-WR networks by relocating data to a time division sub-channels on different wavelength channels. Data $c_{1}$ and $c_{2}$ in Fig. 1 are relocated from wavelength channels $\lambda_{1}$ at input port 1 to $\lambda_{2}$ at output port 1 . Otherwise, data $a, b_{1}$ and $b_{2}$ cannot be switched to their desired time division sub-channels. Wavelength conversion has been heavily investigated since the early proposals of WR networks [1], and technologies have been developed for applications including fast wavelength switching and contention resolution in optical switches [8]. Most time-slot routing schemes in OTDM-WR networks [4]-[7], however, treat wavelength conversion as an option in reducing the blocking probability.

\section{WAVELENGTH SWITCHED TIME-SLOT ROUTING}

\section{A. Routing node architecture}

In this paper, we consider a OTDM-WR network in which each node is assumed to have slot-by-slot wavelength converters and a wavelength-routed optical circuit switch, but no fast optical time-shared space switches. The wavelength converters are installed at each input port of the switch. The timeslot routing relies on fast wavelength convertion instead of fast time-shared space switching. The optical circuit switch routes data according to their associated wavelength only. Figure 2 shows such a wavelength-switched time-slot routing node. In Fig. 2, switch SW has been configured to route data in $\lambda_{1}$ and $\lambda_{2}$ from input port $x$ to output ports $x$ and $y$, respectively, where $x=1,2$ and $y \neq x$. The wavelength converter at input port 1 converts a slot of data into $\lambda_{1}$ if the desired output port is output port 1 , and converts the slot to $\lambda_{2}$ if otherwise. The wavelength converter at input port 2 operates similarly. Hence, the associated wavelegnths of the data slots $b, e$, and $c, d$ are changed from $\lambda_{1}$ to $\lambda_{2}$ and $\lambda_{2}$ to $\lambda_{1}$, respectively. The associated wavelengths of data $a$ and $f$ remain unchanged.

In the proposed routing node architecture, most of the complexity of time-slot routing is in the wavelength converters at the input ports. The number of required wavelength converters is on the order of $\mathcal{O}(K \times W)$ where $K$ and $W$ are the numbers of input/output ports, and the number of wavelengths per fiber, respectively. Like other time division switching node architectures [4], the proposed routing node has internal blocking problem. If a given input/output port pair in a node is not connected by a wavelength channel and all the wavelengths from the input port is exhausted, no wavelength conversion can send a data slot from the input port to the output port. We may reduce or remove the internal blocking by adding extra wavelengths in-

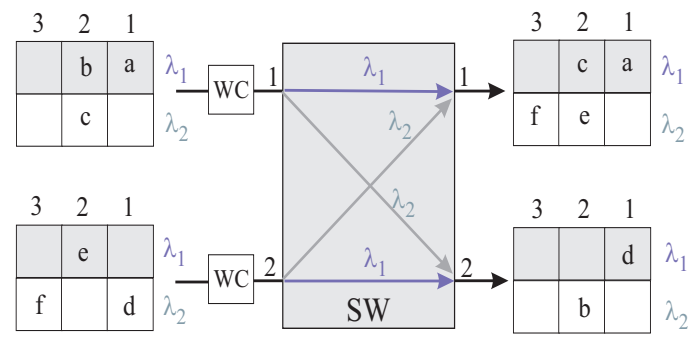

Fig. 2. A wavelength switched time-slot routing node. SW is a wavelength routed optical switch which has been configured to route data in $\lambda_{1}$ and $\lambda_{2}$ from input port $x$ to output ports $x$ and $y$, respectively, where $x=1,2$ and $y \neq x$. WCs are wavelength converters that converts the data slot-by-slot into other wavelengths so that data can be wavelength-routed to the desired output ports.

side the node to provide additional internal routing paths. However, this increases the complexity of the node and requires extra wavelength converters at both the input and output ports. In Fig. 2, output conflict occurs when output port 1 is the desired output port for both data $b$ and $c$. To resolve the conflict, we either block one of the data, reconfigure the node internal paths, or route one of the data using an alternative path if it exists. Reconfiguring the switch internal paths may interrupt existing connections and should be avoided. A dynamic path searching algorithm is therefore important.

\section{B. A shortest path searching algorithm}

Traditional TDM networks assumes time switching and space switching ability at each node. In the proposed OTDMWR networks, we assume that the nodes have fast wavelength converters only, thus traditional shortest path search algorithms [2] must be modified to take into account this constrain. In the following, we assume that i) the path searching algorithm has update information of the whole network, ii) the time required for path searching is negligible when compared to inter-arrival time of users, iii) when two or more shortest paths exist, one of them is chosen at random, and iv) a path may visit a node multiple times provided that the total path length is the minimum. For assumption iv), consider the $6 \times 6$ Manhattan Street Network (MSN) shown in Fig. 3. Assume that each fiber has a single wavelength channel, and the wavelength channels (148-2) and (9-8-7) already exist when we search the shortest path from nodes 14 to 7 . Thus path (14-8-7) is not allowed. Path (14-8-2-3-9-8-7) can be the shortest path under such situation even though it visits node 8 twice.

In the following we describe an algorithm to search the path with the smallest hop count link by link. Although the algorithm assumes that each user requests a single channel only and there is no parallel fibers between nodes, one can easily extend the algorithm to remove the constrains.

We assume that each link is uni-directional. For convenience, we label each link by the input port of the node to which the link is attached. Time division sub-channels are represented by time slots. Let there be $W$ wavelengths per fiber and $T$ time slots 


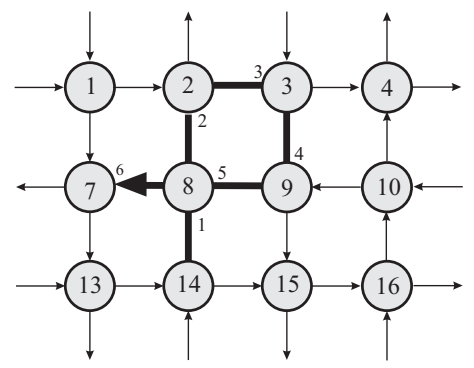

Fig. 3. A possible shortest path in a $6 \times 6 \mathrm{MSN}$ network. Each fiber have a single wavelength channel. Wavelength channels (14-8-2) and (9-8-7) already exist when we search for the shortest path from nodes 14 to 7 .

per wavelength. We first set the weightings of all input ports of the nodes to be infinite. For a given source and destination node pair, the search for the shortest path (in hop count) starts from the input ports of the nodes to which the source node connects. Note that since the links are assumed to be uni-directional, it is not necessary to consider nodes that connect to the source node. We assume that a free time slot at one wavelength channel can be changed to any wavelength channel when it arrives at an input port. Hence, we check the same time slot position, e.g., time slot $x$, in all wavelength channels in the links from the source node to its neighboring nodes. If there is a free time slot in one of the $W$ wavelength channels, the weighting of the input port of the node that the source node connect to is set to 1 . After updating the weightings of all input ports of the nodes that the source node connect to, we choose the input port hence the link that has the lowest weighting. If more than one input ports have the same lowest weighting, one is chosen at random. Let $A$ be the input port of node $N_{A}$ and it has lowest weighting of $W(A)$. For example, we have $A=1, N_{A}=8$ and $W(1)=1$ in Fig.3. We then update the weightings of the input ports of the nodes that $N_{A}$ connects to, e.g., input ports of nodes 2 and 7 in Fig.3. The weightings of the input ports of the nodes that $N_{A}$ connects to will be set to the minimum of the input port original weightings and $(W(A)+1)$ if (1) there already exists wavelength channels with free time slot $x$ in the links connecting $A$ of $N_{A}$ to the nodes, or (2) we can setup new wavelength channels with free time slot $x$ in the links connecting $A$ of $N_{A}$ to the nodes, e.g., the input port 2 at node 2 in Fig.3. Otherwise, there will be no change in the weightings. After updating the input ports of all nodes to which $N_{A}$ connects, we then find the input port with the next lowest weighting from all nodes, not just the nodes to which $N_{A}$ connects. Thus we may return to the nodes to which the source node connects. If one is found, we repeat the above process for this node. This procedure continues until the input port with the next lowest weighting belongs to the destination node or all remaining input ports have infinite weightings. The shortest path can be determined after all alternate paths for all $T$ time slots have been searched.

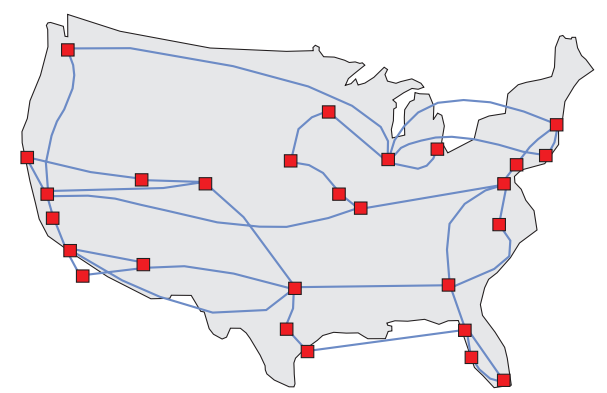

Fig. 4. The AT\&T North America OC-48 fiber network.

\section{PERformance Evaluation}

To demonstrate the proposed time-slot routing scheme, we use discrete event simulations on two different network topologies: a $6 \times 6$ Manhattan Street Network, and the 27-node network of topology of AT\&T North America OC-48 fiber network shown in Fig. 4. In each simulation, new users are randomly generated assuming Poisson probability distribution and they randomly arrive at a node in the network. The destination of the user is chosen from the remaining nodes at random. In the simulations, one time slot will be assigned to each accepted user. If the path searching algorithm cannot find a path, the call is blocked immediately. We set the number of wavelength channels per fiber $W$ and the number of time division sub-channels per wavelength $T$ to the relatively small values of 4 and 20, respectively. Hence, blocking occurs even under low user arrival rate and the performance of the path searching algorithms become important. Once a call is set up, the holding time is an independent exponential random variable with a mean of one time unit. We use the batched mean method to compute the results. The batch size is $10^{4}$ time unit. The first batch result is discarded. The $95 \%$ confidence intervals are less than $5 \%$ of the results.

We simulate the proposed routing scheme in two settings: normal and ideal. The normal setting assumes that the available wavelengths inside the node in Section III-A is exactly equal to the set of wavelengths at the input and output ports of the node. The ideal setting assumes that there are sufficient extra wavelengths inside the node such that no blocking occur inside the node. Two time-slot routing schemes are included for comparison. The first is pure wavelength routing which is the basic routing algorithm in OTDM-WR networks in which there is no time-slot switching or wavelength conversion at intermediate nodes. To search the shortest path in a pure wavelength routed OTDM-WR network, we use the algorithm in Section III-B by setting one wavelength per fiber for all wavelength time-slot combinations. The next is space-switched routing which is similar to the OTDM-WR networks assumed in [4]-[7]. A data slot can be switched from an input port to any output port but the associated wavelength and time division sub-channel remain unchanged. A OTDM-WR network of space-switched routing is equivalent to $W \times T$ identical single channel circuit-switched 


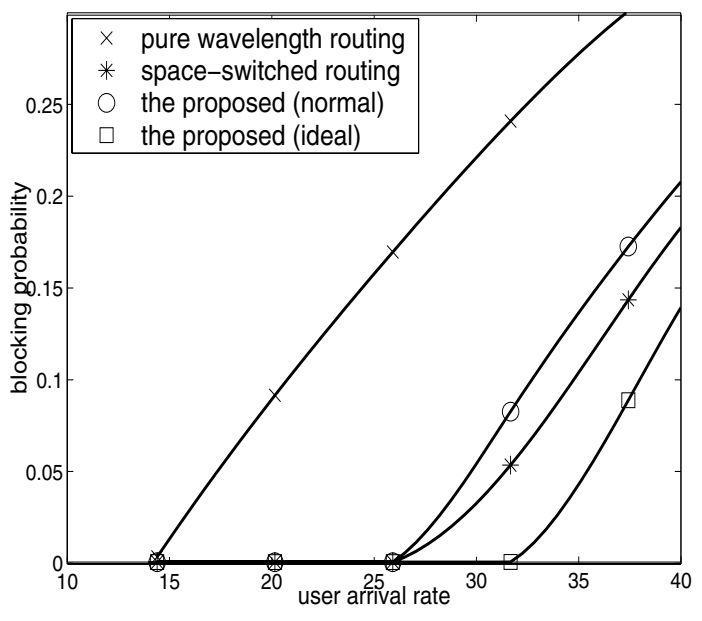

Fig. 5. Blocking probabilities of the routing schemes in a OTDM-WR network with the topology of a $6 \times 6 \mathrm{MSN}$.

networks joining at the source and destination nodes. The shortest path searching in such networks is equal to choosing the 'shortest' path from the set of 'shortest paths' of each of the $W \times T$ equivalent networks.

Figures 5 and 6 show the blocking probabilities of the routing schemes in a $6 \times 6 \mathrm{MSN}$, and the 27-node network of topology of AT\&T OC-48 fiber network shown in Fig. 4, respectively. The $x$-axis is the total number of users arriving in unit time while the $y$-axis shows the average blocking probability of all users. Crosses, asterisks, circles, and squares represent the results of the pure wavelength routing, space-switched routing, the proposed routing scheme in normal and ideal settings, respectively. We observed that at low arrival rate, all four types of routing performed similarly. When the arrival rate increases, the proposed scheme in ideal setting perform better than the time-shared space switched routing which in turn is better than the proposed scheme in normal setting for the MSN and AT\&T networks. The three routing schemes are much better than pure wavelength routing. The two proposed schemes and spaceswitched routing use the alternate paths to prevent blocking at low user arrival rate. This increases the average path lengths and causes network congestion when user arrival rate is high. Since the average node degree of the AT\&T network is larger than that of MSN, more alternate paths are available. Hence, the blocking probabilities shown in Fig. 6 for all the routing schemes at the same user arrival rate are smaller than that shown in Fig. 5.

Figures 5 and 6 demonstrate the flexibility of the proposed scheme. We can optimize the performance of the proposed scheme by adding sufficient extra wavelengths inside the routing node. Or, we may minimize the hardware requirement by using the same number wavelengths inside the node as that at the node input/output ports. In such case, the proposed scheme will have larger blocking probability than that of spaceswitched routing, especially, when the user arrival rate is high.

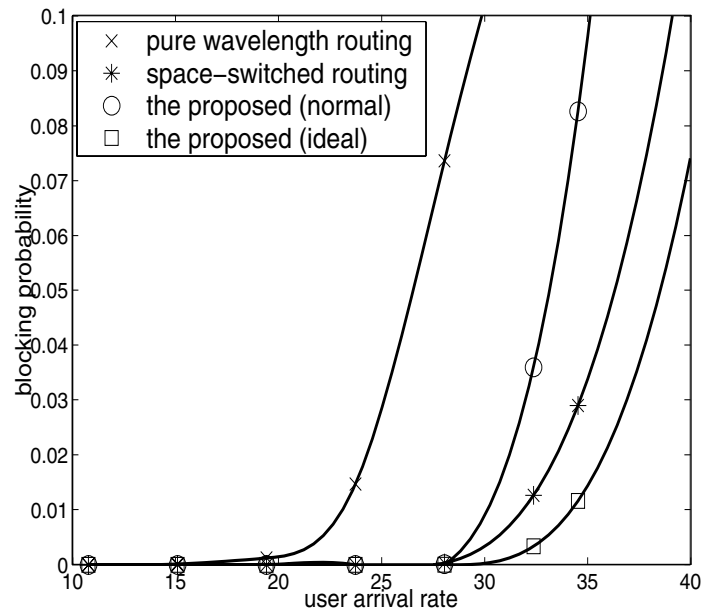

Fig. 6. Blocking probabilities of the routing schemes in a 27-node OTDM-WR network of topology of AT\&T OC-48 fiber network shown in Fig. 4.

\section{CONCLUSION}

We have proposed OTDM-WR networks that can be constructed with fast wavelength converters only. Simulations show that the proposed OTDM-WR networks have much better performance than that of WR networks. When compared to OTDM networks with pure time-shared space switching, the proposed network will have better performance if additional wavelengths are used inside the node to remove internal blocking. Otherwise, the former will have slightly better performance.

\section{REFERENCES}

[1] K.C. Lee and V.O.K. Li, "A wavelength-convertible optical network," Journal of Lightwave Technology, Vol. 11, No. 5/6, pp. 962-970, 2000.

[2] H. Zang, J.P. Jue, and B. Mukherjee, "A review of routing and wavelength assignment approaches for wavelength-routed optical WDM networks," Optical Network Magazine, Vol. 1, No. 1, p.47-60, 2000.

[3] K.L. Deng, R.J. Runser, P. Toliver, I. Glesk, and P.R. Prucnal, "A highly-scalable, rapidly-reconfigurable, multicasting-capable, 100-Gb/s photonic switched interconnect based upon OTDM technology," Journal of Lightwave Technology, Vol. 18, No. 12, pp. 1892-1904, 2000.

[4] N.F. Huang, G.H. Liaw, and C.P. Wang, "A novel all-optical transport network with time-shared wavelength channels," IEEE Journal of Selected Areas in Communications, Vol. 18, No. 10, pp. 1863-1875, 2000.

[5] S. Subramaniam, E.J. Harder, and H.A Choi, "Scheduling multirate sessions in time division multiplexed wavelength-routing networks," IEEE journal of Selected Areas in Communications, Vol. 18, No. 10, pp. 21052110, 2000.

[6] R. Srinivasan, and A.K. Somani, "A generalized framework for analyzing time-space switched optical networks," IEEE journal of Selected Areas in Communications, Vol. 20, No. 1, pp. 202-215, 2002.

[7] "Routing, wavlength and time-slot assignment in time division multiplexed wavelength-routed optical WDM networks," IEEE Infocom 2002, pp. 1442-1450, 2002.

[8] J.M.H. Elmirghani, and H.T. Mouftah, "All-optical wavelength conversion: technologies and applications in DWDM networks" IEEE Communications Magazine, Vol. 38, No. 3, pp.] 86-92, 2000.

[9] S. Pau, J. Yu, K. Kojima, N. Chand, and V. Swaminathan, "160-Gb/s alloptical MEMS time-slot switch for OTDM and WDM application," IEEE Photonics Technology Letters, Vol. 14, No. 10, pp. 1460-1462, 2002.

[10] J.C. McDonald, Fundamentals of Digital Switching, 2nd Edition, Plenum Press, New York, 1990. 